\title{
Adaptation in electric hearing: analysis of level and amplitude modulation encoding
}

\author{
Joshua H Goldwyn*, Eric Shea-Brown \\ From Nineteenth Annual Computational Neuroscience Meeting: CNS*2010 \\ San Antonio, TX, USA. 24-30 July 2010
}

Neural adaptation in sensory systems can potentially improve the efficiency and fidelity of neural encoding (e.g. [1]). In the auditory system, for instance, the firing rates of peripheral and midbrain neurons can shift to better represent the range of most likely stimulus levels $[2,3]$. Adaptation has long been known to exist throughout the auditory system in response to acoustic stimulation, and now studies have revealed that the auditory nerve exhibits firing rate adaptation in response to electric stimulation [4].

Adaptation may, therefore, have important implications for the function and design of cochlear implants, but there have been few attempts to investigate the relationship between neural adaptation, cochlear implant design, and listening outcomes for cochlear implant users. We hypothesize that it will be possible to identify time scales of adaptation that optimally encode a stimulus, depending on the time scales of the stimulus. The time course of adaptation appears to depend on stimulus parameters [5], so if our hypothesis is true, then it may be possible to design stimulation strategies that induce adaptation in a way that improves encoding of cochlear implant stimuli.

To address this hypothesis, we use a point process model to simulate the response of an auditory nerve cell to electric stimulation. The model replicates known features of the auditory nerve's response to electric stimulation including spike threshold, input/output function, and refractory effects. For this study, we implement firing rate adaptation in the model via a spike-history feedback mechanism. The adaptation component has two parameters that control the time scale and degree of adaptation.

We simulate the effect of adaptation on encoding of stimulus level and sinusoidal amplitude modulation. We

\footnotetext{
* Correspondence: jgoldwyn@uw.edu

Department of Applied Mathematics, University of Washington, Seattle, WA 98195, USA
}

use the likelihood function associated with the point process model to quantify spike timing information, as well as common measures of spike count information, strength of phase-locking to the amplitude-modulated signal, and signal-to-noise ratio of firing rates. We find that at high stimulus levels, firing rate adaptation prevents saturation and therefore improves sensitivity to level increments and modulation. In response to weaker stimuli, adaptation typically degrades the encoding of level and amplitude modulation. In general, we find that the degree, not time scale, of adaptation is the more important factor in determining the effect of adaptation on encoding. In order to further probe the effects of the time scale of adaptation, we analyze neural encoding of dynamic stimuli that feature multiple time scales and non-stationary levels.

\section{Acknowledgements}

The authors thank Dr. Jay Rubinstein, Dr. Adrienne Fairhall, and their respective lab groups for numerous helpful discussions. The authors also acknowledge funding support from NIH grant 1F31DC010306-01 (J.H.G.) and a Burroughs-Wellcome Fund Career Award at the Scientific Interface (E.S.-B.).

\section{Published: 20 July 2010}

\section{References}

1. Wark B, Lundstrom BN, Fairhall A: Sensory adaptation. Curr Opin Neurobiol 2007, 17:423-429.

2. Wen B, Wang Gl, Dean I, Delgutte B: Dynamic range adaptation to sound level statistics in the auditory nerve. J Neurosci 2009, 29:13797-13808.

3. Dean I, Harper NS, McAlpine D: Neural population coding of sound level adapts to stimulus statistics. Nat Neurosci 2005, 8:1684-1689.

4. Zhang F, Miller CA, Robinson BK, Abbas PJ, Hu N: Changes across time in spike rate and spike amplitude of auditory nerve fibers stimulated by electric pulse trains. J Assoc Res Otolaryngol 2007, 8:356-372.

5. Woo J, Miller CA, Abbas PJ: The dependence of auditory nerve adaptation on electrical stimulus parameters, electrode position, and fiber diameter: a computer model study. J Assoc Res Otolaryngol 2009, DOI 10.1007/s10162-009-0199-2 (online ahead of publication).

doi:10.1186/1471-2202-11-S1-P166

Cite this article as: Goldwyn and Shea-Brown: Adaptation in electric hearing: analysis of level and amplitude modulation encoding. BMC Neuroscience 2010 11(Suppl 1):P166. 